\title{
Establishing Laboratory Cultures and Performing Ecological and Evolutionary Experiments with the Emerging Model Species Chironomus Riparius
}

Author names and affiliations:

Quentin Foucault ${ }^{1,2}$, Andreas Wieser ${ }^{1,2}$, Ann-Marie Waldvogel ${ }^{1}$, Markus Pfenninger ${ }^{1,2}$

1 Molecular Ecology Group, Senckenberg Biodiversity and Climate Research Centre, Senckenberganlage 25, 60325 Frankfurt am Main, Germany

${ }^{2}$ Johannes Gutenberg University Mainz, Institute of Organismic and Molecular Evolution, Johann-Joachim Becher-Weg 7, 55128 Mainz

Corresponding author: QF, quentin.foucault@ senckenberg.de; phone +33(0)603221891

Email addresses:

QF: quentin.foucault@senckenberg.de

AW: andreas.wieser@senckenberg.de

AMW: ann-marie.waldvogel@senckenberg.de

MP: markus.pfenninger@ senckenberg.de

\section{Abstract}

Chironomus riparius is a well-established model organism in various fields such as ecotoxicology and ecology, and therefore environmental preferences, ecological interactions and metabolic traits are well-studied. With the recent publication of a high-quality draft genome, as well as different population genetic parameters such as mutation and recombination rate, the species can be used as an alternative to the Drosophila models in experimental population genomics or molecular ecology. To facilitate access to this promising experimental model species for a wider range of researchers, we describe experimental methods to first create and sustain long term cultures of $C$. riparius and then use them to perform repeatable and comparable experiments for various research questions.

Keywords : Chironomus riparius, laboratory cultures, experimental evolution 


\section{INTRODUCTION}

Studying model organisms enabled scientists to test and establish major theories in life sciences. This is possible through extensive knowledge collected on these organisms over decades, at all levels of investigations (e.g. behaviour, phenotype, metabolism, genetics, etc.). Routinely, this knowledge is transferred to other organisms to model and predict their possible responses in various scenarios, as e.g. when their testing is not possible due to financial, technical or logistical infeasibility or ethical reasons. However, generalising responses of few model organisms comes necessarily with a loss in accuracy at best, and might even lead to outright wrong inferences. Increasing the number and diversity of study organisms, and thus the range of life cycles, demography and environmental responses, could alleviate this effect and allow drawing generalised conclusions. For this purpose, it is necessary to establish and share knowledge about sampling, reliable identification, rearing, and experimental procedures of diverse organisms to increase our detailed knowledge of biodiversity.

Chironomids are already well-established model organisms in ecotoxicological studies (Watts and Pascoe 1996, Ha and Choi 2008) as well as bio-indicators for ecology (De Bisthoven et al. 2005), paleoclimatology (Brooks and Birks 2001) and paleoecology (Walker 1987). For this reason, we possess profound knowledge of their responses to a broad range of environmental or anthropic constrains such as pollution (Williams et al. 1986, Taylor et al. 1991) or climate change (Quinlan et al. 2005, Nemec et al. 2013)

In this manuscript, we describe methods to start, raise and maintain permanent laboratory populations of Chironomus riparius (Meigen, 1804), and to estimate different important life history parameters of those populations. This approach aims at sharing methods and allowing easy replication to increase the gathering of data on this species but also on ecologically similar taxa in natura. On the long term, this permits the creation of a knowledge base that could help 
testing innovative hypotheses on both, the ecological and evolutionary level. C. riparius, also known as the harlequin fly, is a multi-voltine non-biting midge with a Holarctic geographic distribution. During its aquatic larval stage, the species lives in muddy substrate of small streams or ponds (Pinder 1986, Armitage et al. 1995), making up an important part of macrozoobenthic communities. Free haemoglobin in their haemolymph stains the larvae prominently red (Neumann 1962), and together with their high abundance in suitable habitats, larvae can be found and sampled easily. Our work builds on the extensive long-term evolutionary ecotoxicology studies performed by Nowak and Vogt (Nowak et al. 2007a, Vogt et al. 2007a, Vogt et al. 2007b, Vogt et al. 2007d).

Moreover, the integration of experimental investigations with genetics and genomics is possible since the publication of a high-quality draft genome as reference sequence (Oppold et al. 2017c). Furthermore, important population genetic parameters have been studied, such as the species-specific spontaneous mutation rate (Oppold and Pfenninger 2017a), its genomic basis of niche differences (Schmidt et al. 2013), demography in European populations (Waldvogel et al. 2018), and its genomic architecture (Kraemer and Schmidt 1993, Zampicinini et al. 2004, Oppold et al. 2017b). The possibility to easily obtain natural populations from a large distribution range, its capacity to cope with laboratory culture conditions, and the availability of genetic and genomic resources makes $C$. riparius a suitable study organism for many disciplines and research areas.

\section{FROM THE FIELD TO THE LAB}

\section{1) Habitat}

C. riparius larvae are commonly found in muddy part with high organic content in small streams, or more rarely in drainage ditches, ponds or long-lived puddles in the Northern hemisphere (Hooper et al. 2003). To select a good location for sampling C. riparius, streams 
located in mixed forests, close to wastewater treatment plants or livestock can be considered as suitable because of the high inflow of organic matter. Agricultural areas have been found less suitable for $C$. riparius despite the presence of organic matter. This is probably mainly due to soil washed into the river by the erosion of agricultural fields, preventing the formation of organic mud, as well as run-offs of insecticides and nitrogen from agricultural fields.

\section{2) Collection and Sampling}

Sampling of larvae is principally possible throughout the year, however, due to massive emergence events; larvae of identifiable size can be periodically scarce or even absent. Sampling is therefore most promising in early fall, when population density is highest, and during late winter, ensuring a maximal size of the larvae which (slowly) grew during the whole winter, before the actual temperature rise of spring leads to emergence (Goddeeris et al. 2001).

Specimens are sampled in the larval stage by using a sieve (appliance kitchen sieve with a mesh size of not more than $1 \mathrm{~mm}$ ) to extract them from the mud. In streams, larvae are more likely found in areas with reduced current, where thick layers of organic mud are building banks. Those places are more likely to contain larvae because of their poor ability to swim against the current. However, muddy stream banks are usually difficult to reach either because of the water level, the current of the stream, the steepness of the riverside or obstructing vegetation. The alternative, wading in the stream to reach the promising sampling site, may be dangerous and can easily destroy it. To reach sampling sites from a safe stand in some distance, a telescopic pole (e.g. from an apple picker with an approximate length of $4 \mathrm{~m}$ ) can be attached to the metal sieve. The sieve should be carefully drawn through the uppermost few centimetres of the mud bank. The mud in the sieve is subsequently washed away by gently shaking it in the downstream current in the river. The bright red Chironomus larvae can then be collected from the washed samples using storkbill fine pointed forceps. With some experience, it is possible 
to directly identify the so-called "thummi" type larvae to which, amongst others, C. riparius belongs (Figure 1). However, final identification, in particular distinction from the cryptic sister species $C$. piger necessarily involves DNA barcoding (see below).

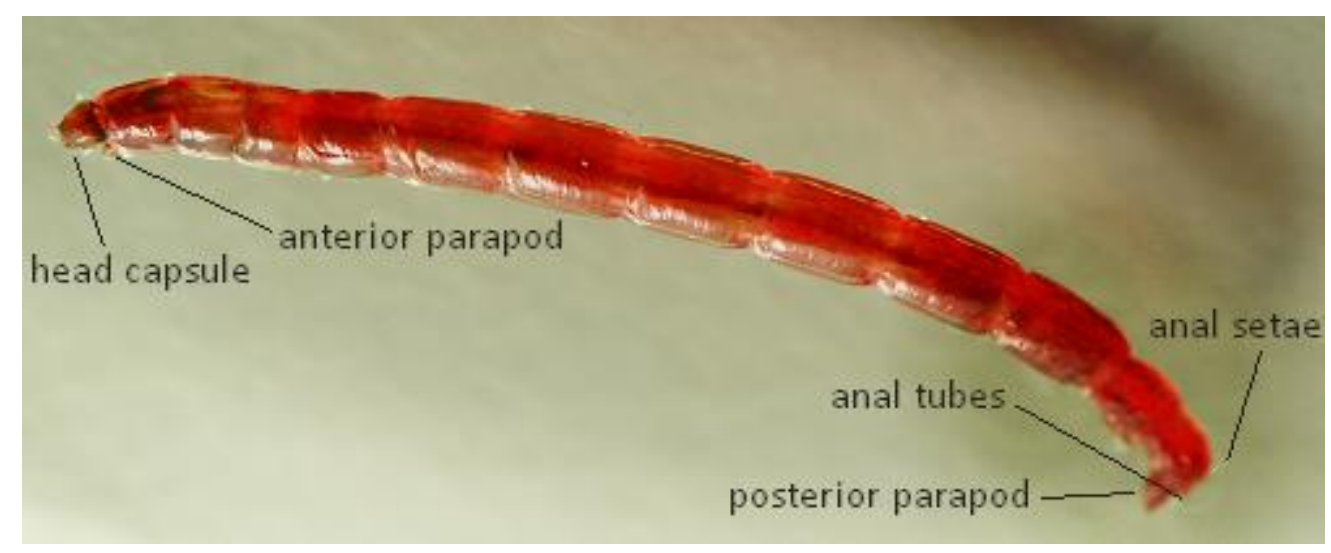

Figure 1: Photograpy of a C. riparius larva.

The visually sorted larvae based on their similarity to the so called thummi larval type (fig1) are then brought back alive to the lab. To ensure that the sampled larvae survive the transport, they are best put in $1 \mathrm{~L}$ beakers (roughly 100 individuals per beaker), containing some washed sand. In order to minimize stress, it is advisable to fill the beaker with water from the sampling site. If transport is expected to take more than a couple of hours, regularly opening the lid to allow aeration or aeration with a battery powered pump, storing them in an insulated container to prevent strong temperature fluctuations, and offering a food source (powdered Tetramin, see below) can minimize the loss of specimen.

To set up a monospecific laboratory population, larvae brought back from the field are directly put in a reproduction cage (see below) where they can complete their larval development, pupate and emerge as adults, swarm and deposit egg clutches. These egg clutches need to be collected daily for species identification via DNA-barcoding. A small piece of each egg clutch is cut off and used for DNA extraction (see below). The remainder of the egg clutch is kept separately in an individually labelled and aerated glass beaker, filled with washed sand and 
medium (see below). For keeping living Chironomus specimen for a longer time-span in a vessel, it is generally advisable to use glass vessels, because i) plastic may contain plasticizers with hormone effects (Mathieu-Denoncourt et al. 2015) and ii) glass vessels can be hygienically cleaned to avoid infections with bacteria and fungi. The use of laboratory grade glass is not necessary, as long as the glass can be cleaned in a dish washer. The extracted DNA of each egg clutch is meanwhile used for DNA barcoding to reliably identify clutches of $C$. riparius or another species of interest (e.g. the sister species C. piger). If it is foreseeable that the DNA barcoding procedure will take longer than a few days, the egg-clutches can be stored at $4^{\circ} \mathrm{C}$ to stall their development. The positively identified egg clutches of the desired species can then be pooled to start a laboratory population or to reinforce an existing one.

The level of genetic variability in the resulting lab population strongly depends on the number of egg clutches used to start the population (Nowak et al. 2007b). If the genetic variability is supposed to approach conditions in the field, not less than 20-30 egg clutches should be used. Please note that mixing egg clutches or reinforcing an existing lab population from different populations may result in diminished fitness due to outbreeding depression among geographically distant populations (Oppold et al. 2017b).

In case no laboratory experiments are desired, larvae can be fixed in $>95 \%$ ethanol directly in the field and then prepared for DNA barcoding in the laboratory.

3) Species identification with DNA-barcoding

To reliably identify $C$. riparius and separate individuals from the cryptic sister species $C$. piger as well as potential hybrids (Pedrosa et al. 2017), the combination of mitochondrial COI barcoding with sequencing of the nuclear marker L44 is necessary. 
For DNA isolation, we apply the HotSHOT procedure (Montero-Pau et al. 2008) on a small piece of the larval tail (the last abdominal segment with posterior parapods and anal tubules, (Fig. 1)). The CO1 mitochondrial locus (forward primer: 5'TCGAGCAGAATTAGGACGACC, reverse primer: 5'-GGATCACCCCCACCAGCAGG)

(Pfenninger et al. 2007) was amplified using a Bio-Rad T100 Thermal Cycler. The reactions were set-up for 35 amplification cycles $\left(95^{\circ} \mathrm{C}\right.$ for $30 \mathrm{~s}, 48^{\circ} \mathrm{C}$ for $1 \mathrm{~min}, 72^{\circ} \mathrm{C}$ for $\left.1 \mathrm{~min} 30\right)$ with an initialization of $5 \mathrm{~min}$ at $95^{\circ} \mathrm{C}$ and a final elongation at $72^{\circ} \mathrm{C}$ for $10 \mathrm{~min}$. Likewise, the L44 nuclear locus was amplified (forward primer: 5'-TGTACGCTTTTGTGCAATC, reverse primer: 5'-TCTCAACACCGACTGATC) using 35 amplification cycles $\left(95^{\circ} \mathrm{C}\right.$ for $30 \mathrm{~s}, 50^{\circ} \mathrm{C}$ for $1 \mathrm{~min}, 72^{\circ} \mathrm{C}$ for $1 \mathrm{~min} 30$ ) with an initialization of $5 \mathrm{~min}$ at $95^{\circ} \mathrm{C}$ and a final elongation at $72^{\circ} \mathrm{C}$ for $10 \mathrm{~min}$ (see Oppold et al. (2016) for details).

The rest of the larvae with the taxonomically relevant head capsule (Epler 2001) can be conserved for documentation or subsequently be used e.g. for genomic analyses (conservation in ethanol at $-20^{\circ} \mathrm{C}$ for short term storage or $-80^{\circ} \mathrm{C}$ for long term storage).

\section{LABORATORY POPULATIONS}

1) Swarming and reproduction

For reproduction, $C$. riparius has a swarming behaviour. Males form a dense swarm to attract females (Caspary and Downe 1971). Usually, males emerge two days before females (Day et al. 1994), so that the latter can immediately fly into the male swarm for copulation. (Downe and Caspary 1973) identified some triggers of the swarming behaviour: light intensity, surface colour and reflection beneath the swarm. The preferred surface referred as "landmark", is described as black and shiny, which is plausible given the muddy water where $C$. riparius are likely to be found in the wild. The fertilised egg clutch is subsequently oviposited attached to 
some solid substrate directly beneath the water surface. Females usually mate with a single male; however, it is important to ensure sufficient space for the swarming of a large population. We therefore perform reproduction in cages of at least $50 \mathrm{~cm}^{3}(1 \mathrm{mx} 1 \mathrm{mx} 50 \mathrm{~cm})$ with a sediment filled water bowl for oviposition at the bottom. However, the swarming behaviour is quite flexible, varying among individuals and could thus also be performed in smaller cages and small swarms (Caspary et al. 1971). It is indeed possible to successfully mate a single male with a single female (Oppold et al. 2017a), however, the success rate is much lower than in a swarm.

Literature reports possible oviposition without female fecundation (Caspary et al. 1971). We confirmed this experimentally by separating larvae during pupal stage and raising the adults individually. This resulted in $35 \%$ of females laying at least one egg clutch without fecundation, including 5\% laying two egg clutches. The majority of egg clutches $(71 \%)$ were initially indistinguishable from regularly fecundated ones. However, the rest contained unusually small eggs (6\% of clutches) or were abnormally formed, resulting in an unorganised egg mass (23\% of clutches). None of the eggs laid by unfecundated female developed to hatching and finally ended up rotting and therefore, parthenogenesis can be excluded.

After the emergence of the first adults of an experimental population at $20^{\circ} \mathrm{C}$, individuals swarm for a period of about 20 days. However, due to individual variation in developing time, swarming may take between 15 and 32 days. During this period, eggs are produced continuously. In a continuous culture, this leads eventually to an overlap of generations increasing with the number of generations (Fig 2). This effect is more pronounced at lower temperatures where the developmental rate is slower. 


\section{Generation Overlap in Lab Populations at $20^{\circ} \mathrm{C}$}



Figure 2: Projection of four C. riparius generations overlapping in laboratory conditions based on the emergence time at $20^{\circ} \mathrm{C}$.

\section{2) Laboratory cultures set -up}

Different methods for cultures of chironomids have already been developed such as the method of (Credland 1973), advising to raise larvae using sediment composed of nettle powder or cellulose from which larvae can directly feed or the method of (Dettlaff and Vassetzky 1990) advising to not raise the midges under water but in wet mud to maximise the oxygen input and thus increasing the developmental rate. While these methods certainly work, they have the problem that i) larvae are not visible and thus optical control of their health and development is difficult, ii) the high organic content is prone to decomposition by bacteria and fungi, which may compromise larval health and iii) regular maintenance (in particular exchange of sediment) of continuous cultures is complicated. 
For laboratory cultures, we hence propose a modified version of the culture method described in the OECD guideline $\mathrm{N}^{\circ} 219$ (OECD 2004) -235 (OECD 2011). It minimises the necessary maintenance work while offering a growth rate suitable for experiments. The cultures are setup with a light-dark rhythm of $16: 8 \mathrm{~h}$ and $60 \%$ humidity. Larvae are raised in an artificial medium, consisting of purified, deionised water adjusted to a conductivity of $520-540 \mu \mathrm{S} \mathrm{cm}^{-}$ ${ }^{1}$ with sea salt (e.g. TropicMarin ${ }^{\circledR}$ ), and a basic pH around 8. Instead of the more expensive cellulose or nettle powder sediments described in previous protocols (Credland 1973), we use washed ( $\mathrm{pH}$ neutral) playground sand that can be cheaply purchased in wholesale. This has the additional advantage of avoiding its frequent replacement and hence a major disturbance for the populations. Permanent cultures are fed daily with 0,4g finely grounded fish food (e.g. Tetramin $^{\circledR}$ Flakes) using an aquarium automatic feeder (e.g. Eheim ${ }^{\circledR}$ Twin Auto Tropical Aquarium Fish Feeder). Contrary to former belief that adults are not feeding because of non functional mouth parts, it has been proven that adult $C$. riparius can indeed feed on sucrose (Goff 1972, Burtt. et al. 1986, T. et al. 1986). This nutrient intake positively impacts their life cycle by increasing reproduction and longevity (Weltje et al. 2009, OECD 2010).

To set up long term cultures, we use climate chambers in which temperature and humidity can be controlled. Instead of climate cabinets, climate chambers provide the necessary space needed for the cage as well as for experimental or maintenance manipulation. We thus recommend the use of at least one square meter of space per experimental population to insure sufficient space for handling both culture and experimentation. However, in the absence of climate chambers any room with an approximately constant environment is suitable to maintain a culture. Note, that if external daylight reaches the room, the population might synchronise to seasons and suffer from fluctuations in productivity. Climate chambers should be furnished with a shelf, neon light with a timer (fig 3-5) and air pumps or an alternative air pressure system (fig 3-4). Cages of $50 \mathrm{~cm}^{3}$ are equipped with an automatic feeder (fig 3-1), 2 plastic trays 
$(40 \times 35 \times 8 \mathrm{~cm})$ filled with sand and medium (fig 3-2) as well as a glass with sugar water $(100 \mathrm{~g} / \mathrm{L})$ for the adults feeding (fig 3-3) in which wooden tongue depressors are placed to permit the individuals to feed without drowning (fig 3-3).

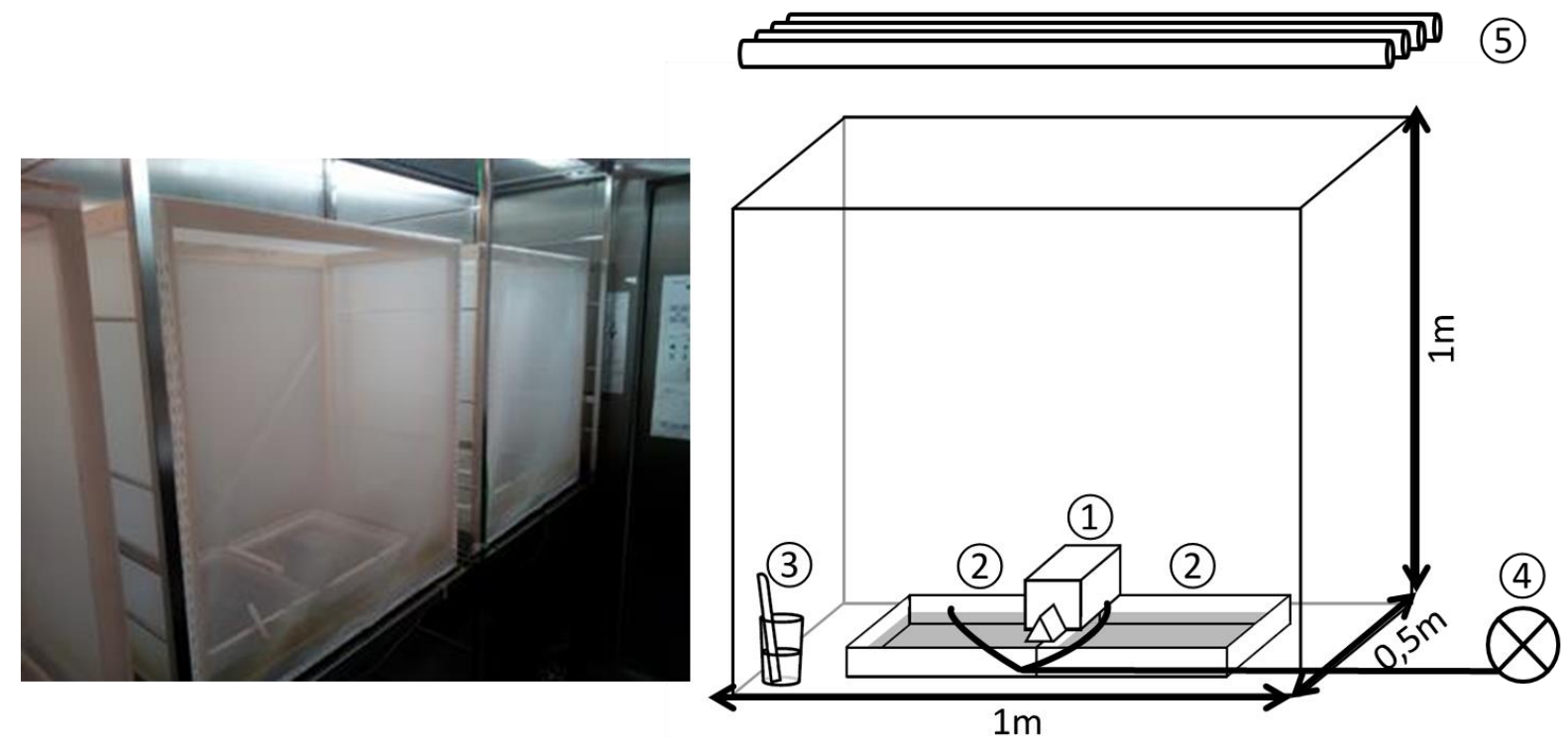

Figure 3: Blueprint and picture of $C$. riparius swarming cages. The numbers on the blueprint designate the following equipment: 1) automatic feeder dispensing $0,4 \mathrm{~g}$ of food per day and tray, 2) plastic trays $(40 \times 35 \times 8 \mathrm{~cm})$ prepared with sediment and medium, 3) sugar water glass with a wooden tongue depressor, 4) aquarium air pump 5) neon light moisture-proof and 16:8 h timer controlled.

\section{3) Maintenance of laboratory populations}

To ensure long term survival of laboratory populations, regular maintenance cycles need to be realised. Our set-up has been optimised to reduce the maintenance cycle duration and -rate to a short bi-weekly maintenance and a more complete monthly maintenance. For the bi-weekly maintenance, three steps are required: (1) refilling of water-levels with deionised water to keep physico-chemical parameters of the medium stable; (2) replacement of the sugar water for the adults, to avoid mould growth; (3) visual control of the electric equipment (e.g. automatic feeder, air pump, light) and presence of left-over food in the culture. Accumulating food in the culture is an indicator of a decrease in population size and can also lead to mold formation and 
risks the death of the culture. If a food deposit accumulates, it is necessary to adjust the food amount or even stop the feeding until all food disappears from the sediment. The rate of the three maintenance steps depends also on the exposure temperature; higher temperatures enhance mold formation requiring more frequent check-ups.

Once per month, a thorough cleaning of the cage is necessary to avoid diseases or contamination. This involves removal of dead individuals and any leftover of food from the bottom of the cage next to the tray using a vacuum cleaner modified to aspire through a PVC hose $(4-6 \mathrm{~mm})$. That way proliferation of collembolans and other insects that could compromise genetic analysis can be avoided.

\section{EXAMPLES FOR EXPERIMENTAL SET-UPS}

\section{1) Experimental protocols}

a) Mutation rate estimations

The estimation of spontaneous mutation rates per generation requires the identification of rare de novo mutations out of a large number of nucleotides with an amount at least a multiple of the species' genome-size. We, therefore, developed the approach of short-term mutation accumulation (MA) lines for $C$. riparius (Oppold et al. 2017a) by combining classical MA line experiments (Mukai and Cockerham 1977, Keightley et al. 2009) with the parent-offspring (trios) scenario (Keightley et al. 2013). Hereby, all full sib individuals of a single egg clutch are used to start separate MA lines. The pooled genomes of all individuals from the egg clutch represent the parental genotypes building the reference level according to the trio approach. The (at least ten) separated MA lines are then inbred for at least five generations to allow for a sufficient number of meiotic turnovers in relation to the genome size that in turn will accumulate enough mutations for an accurate rate estimate. In calibration to the parental genotypes as reference genome-pool, each MA line serves as experimental replicate to allow 
for an accurate estimate of the spontaneous mutation rate. Further details on the experimental design can be found in Oppold et al. (2017a).

b) Experimental evolution

Experimental evolution studies allow the investigation of long term effects of selection pressures (Merilä and Hendry 2014). what is perfectly possible with C. riparius populations. For such long-term experiments, replicate populations with 2000 individuals are created, originating from 200 different eggs clutches. The latter are randomly sampled to ensure sufficient genetic diversity (Nowak et al. 2007b). Afterwards, those populations are split in half in two identical plastic trays $(40 \times 35 \times 8 \mathrm{~cm})$ prepared with medium and sediment to avoid problems related to larval over-crowding. The two trays are then placed in cages as described above. For these experiments, we ignore overlapping generations since this process is happening in natural populations and it allows avoiding disturbance by manipulation inside the cage. If, however, non-overlapping populations are essential for the experimental design, it is possible to remove the egg-clutches produced during a generation from the culture, synchronise them by keeping them at $4^{\circ} \mathrm{C}$ until sufficient egg-clutches were laid and transfer them to a new cage. This cold treatment is not recommended for experiments related to temperature effect due to hardening effect that could be triggered by this treatment (Lee et al. 1987, Kelty and Lee 2001). In this case, egg-clutches have to be transferred every day to a new cage during the whole swarming period to isolate generations. This isolation would permit them to lay eggs in a medium that is free of larvae from the previous generation but will create other bias such as the exclusion of late emerging larvae.

c) Life-cycle fitness test

To monitor overall population fitness, we estimate the population growth rate (PGR) in a standardised Life-cycle Fitness Test (LCFT) (OECD 2004, OECD 2010, OECD 2011, 
Waldvogel et al. 2018) with modifications. A group of individuals of the same population is exposed to the parameters or stressors of interest over their entire life-span. Each experimental block comprises usually five replicates coming from the same five egg clutches, belonging to the same population to exclude any "family" effect. Each replicate consists of 30 larvae, summing up to a total of 150 larvae for each experimental block. The replicates for one block are prepared by randomly sampling larvae from egg clutches freshly hatched on the same day. The larvae are then placed in glass bowls $(20 \mathrm{~cm}$ of diameter and $10 \mathrm{~cm}$ of height), prepared with medium and sand as described above. The replicates are raised in the bowls covered with nets during the whole larval stage with a daily feeding calculated for the respective


and $0.5 \mathrm{mg} \cdot$ larvae $^{-1}$ for the L4 stage). The emerged individuals are then collected using a custom aspirator, made of a $50 \mathrm{ml}$ falcon tube equipped with a mouthpiece at one side, composed of a hose (diameter $\sim 10 \mathrm{~mm}$ ) and a filter made of gauze. At the other extremity of the falcon tube, a sampling hose (diameter $\sim$ ) is attached through which the adults are sucked. The sex as well as the emergence day of the sampled imagines is recorded to calculate larval mortality as the number of larvae that did not reach the pupation stage. All adults from each replicate are placed together in a single reproduction cage and the eggs produced are collected and counted. The number of eggs in each clutch is estimated using a modified version of the method described by Sibley et al. (1997). It consists of counting the number of spires of the clutch and the number of eggs present in three of the spires (one of each end of the egg clutch and one in the middle) to get a mean. The fertility is then measured as the number of egg clutches where at least some eggs hatched. After recording the fertility and the estimated number of eggs per clutches, we apply a simplified Euler-Lotka calculation to obtain the PGR:

$$
P G R=\left[(j \times i \times h) \times\left(1-\frac{f}{100}\right)\right]^{\frac{1}{g}}
$$


where (f) is the mortality rate, $(\mathrm{g})$ the mean emergence time of females, (h) the female fraction , (i) the number of eggs per egg mass and (j) the number of fertile egg masses per female (Vogt et al. 2007c). The population growth rate allows us to compare the effects of experimental treatments on the demography and thus inclusive fitness of the population, linking ecological with evolutionary effects.

d) Measuring phenotypic plasticity

To investigate the origin of plastic or genetic phenotypical modification caused by some factor, we designed a common garden experiment permitting the exposure of offspring of a population to different intensities of any factor. For this set-up, two intensities that which the parental generation had never experienced before (one lower and one higher) were used as experimental treatments, whereas the intensity actually experienced by the parental generation serves as control. For this experiment, we used mortality as response factor for inferring phenotypic modifications caused by the experienced factor, but any measurable response as well as combination of several could be used. Five egg clutches per replicate were hatched and larvae from those egg clutches were sampled and isolated in 6 wells plates for $48 \mathrm{~h}$, i.e. one larva per well. The mortality is then measured after $24 \mathrm{~h}$ and again after $48 \mathrm{~h}$ by monitoring movement of the larvae under a suitable binocular. After $48 \mathrm{~h}$, all surviving larvae were put in glass bowls prepared with sediment and medium as described above. During this step, larvae originating from the same egg clutch are pooled and raised, still exposed to the same intensity of the factor, during all the larval stages with same feeding protocol as for the LCFT (see above). The day of emergence of the adults as well as the final mortality is recorded and compared between the different factors. Mortality data obtained through this experiment will indicate if phenotypical plasticity is the major way to deal with the factor depending if the mortality was lower in the intensity experienced by the parents or the same at all intensities. 
e) Hybridisation experiments

To investigate the hybridisation rate between $C$. piger and $C$. riparius, we performed mating experiments using laboratory cultures. Experiments were carried out in climate chambers with $60 \%$ humidity and a 16:8h light/dark rhythm. Five eggs clutches were sample for each of the monospecific cultures and were put to hatch. From those, 6 replicates of 50 larvae for each culture were raised in glass bowls until emergence using the protocol explained in section 3.1.c. Pupae were sampled daily and isolated in six well plates until emergence to avoid preexperiment reproduction. Emerging adults were then transferred in reproduction cages prepared as in section 2.2 and forced to hybridise by combining males of one specie with the females of the other species in each cage and vice versa. Egg clutches from those crossings were sampled and synchronized by placing them at $4{ }^{\circ} \mathrm{C}$. The protocol was repeated using the egg clutches from the initial crossings to finally obtain every F2 crossing and then for every possible backcrossing. The population growth rate of each of these crossings has been determined using the protocol described in section 3.1.c to estimate the viability of each crossing directions.

\section{2) Set-up for parameter determinations}

For modelling purposes, life history and physiological traits often need to be parametrised. For this purpose, we designed experiments to experimentally determine parameters from experimental or natural populations.

a) Experimental determination of daily mortality rate in adult chironomids

To determine the daily mortality rate of adults, cultures are monitored for one generation in a constant environment to allow for acclimatisation. From these cultures, 20 pupae per replicate are sampled during a short time period to ensure that all individuals emerged at approximately 
the same time. The pupae are then placed in a prepared cup (fig4). The number of individuals per cup is limited to 20 pupae per cup to avoid crowding effects. After $24 \mathrm{~h}$, we discarded the non-hatched, remaining pupae and documented the number of emerged adults. To obtain the desired parameter, we documented the number and sex of dead individuals every day. As sex determination of dead individuals might be difficult, the sex of each individual has to be documented at the beginning of the experiment. This experimental set-up has been performed at different temperatures to infer the relation between ambient temperature and expected life span.
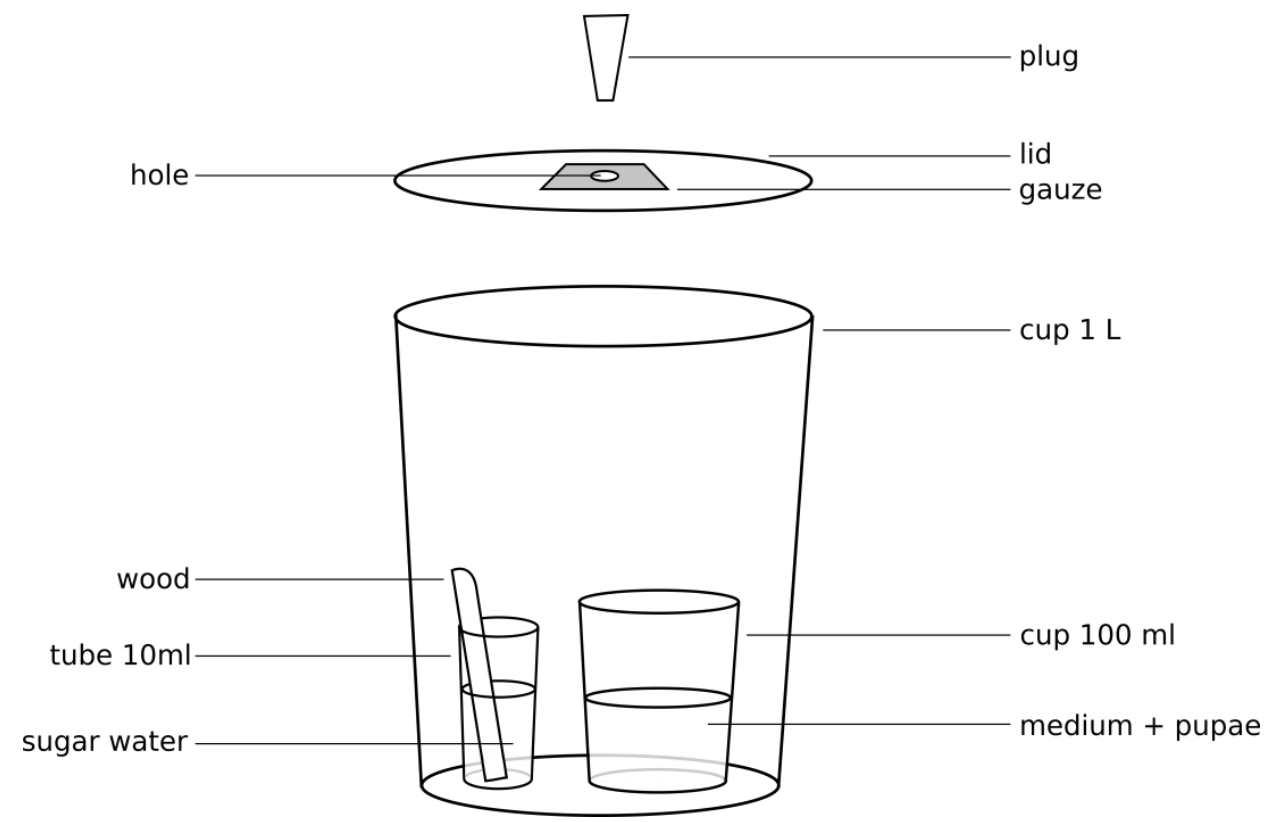

Figure 4: Experimental mortality cup blueprint.

b) Determination of average time to oviposition

With this experiment, we aimed to determine the average time between emergence and oviposition. This is done by placing newly emerged adult chironomids in a swarming cage, containing a bowl of water as vessel for oviposition and a glass of sugar water as food, as described above. Adult individuals emerged within the last $24 \mathrm{~h}$ are transferred by simply placing the vessel a swarming cage for $24 \mathrm{~h}$. After the adults emerged in the cage, the number 
of deposited egg-clutches is documented every day. The number of females in the cage is monitored daily and the experiment is terminated when the last female has died.

\section{CONCLUSION}

All protocols described above are either refinements of official OECD guidelines or have been explicitly developed through years of experiments on C. riparius. Procedures were simplified to be easily performed and highly sustainable through time with the least maintenance possible, making them highly reproducible and suitable for long-term experiments with reduced personnel involved. However, the simplifications created also some bias as trade-off for reducing the manipulations and so the disturbance of the populations during the experiments. Thereby, unlike the previous methods of C. riparius populations rearing (Credland 1973), our set-up does not include any change of sediment or medium of the cultures, leading to a steady increase of the conductivity of the medium by the input of organic matter compromising the stability of the environment. Such kind of increase, even if it has slight or even no effect on the survival of $C$. riparius, has to be monitored through time and included in the analysis to avoid false positives at the genomic or phenotypic level. Many of those experiments bear the advantage of being easily reproduced and even if many have been performed with temperature as treatment factor, they can be easily modified to test a wide range of abiotic conditions on populations of $C$. riparius increasing the repeatability and comparability of results for future studies.

\section{References}

- Armitage, P., P. Cranston and L. Pinder (1995). The Chironomidae: the biology and ecology of non-biting midges Chapman and Hall. London, UK 
- Brooks, S. J. and H. Birks (2001). Chironomid-inferred air temperatures from Lateglacial and Holocene sites in north-west Europe: progress and problems. Quaternary Science Reviews 20(16-17): 1723-1741

- Burtt., R. J. O. Perry and A. J. McLachlan (1986). Feeding and sexual dimorphism in adult midges (Diptera: Chironomidae). Ecography 9(1): 27-32 doi: doi:10.1111/j.16000587.1986.tb01188.x.

- Caspary, V. and A. Downe (1971). Swarming and mating of Chironomus riparius (Diptera: Chironomidae). The Canadian Entomologist 103(3): 444-448

- Credland, P. F. (1973). A new method for establishing a permanent laboratory culture of Chironomus riparius Meigen (Diptera: Chironomidae). Freshwater Biology 3(1): 45-51

- Day, K., R. S. Kirby and T. Reynoldson (1994). Sexual dimorphism in Chironomus riparius (meigen): Impact on interpretation of growth in whole-sediment toxicity tests. Environmental Toxicology and Chemistry: An International Journal 13(1): 35-39

- De Bisthoven, L. J., A. Gerhardt and A. Soares (2005). Chironomidae larvae as bioindicators of an acid mine drainage in Portugal. Hydrobiologia 532(1-3): 181-191

- Dettlaff, T. A. and S. G. Vassetzky (1990). Animal Species for Developmental Studies, Springer US.

- Downe, A. and V. Caspary (1973). The swarming behaviour of Chironomus riparius (Diptera: Chironomidae) in the laboratory. The Canadian Entomologist 105(1): 165-171

- Epler, J. H. (2001). Identification manual for the larval Chironomidae (Diptera) of North and South Carolina. A guide to the taxonomy of the midges of the southeastern United States, including Florida. Special Publication SJ2001-SP13. North Carolina Department of 
Environment and Natural Resources, Raleigh, NC, and St. Johns River Water Management District, Palatka, FL 526

- Goddeeris, B. R., A. C. Vermeulen, E. De Geest, H. Jacobs, B. Baert and F. Ollevier (2001). Diapause induction in the third and fourth instar of Chironomus riparius (Diptera) from Belgian lowland brooks. Archiv für Hydrobiologie 150(2): 307-327

- Goff, A. (1972). Feeding of adult Chironomits riparius Meigen. Mosquito News 32(2): 243244

- Ha, M.-H. and J. Choi (2008). Effects of environmental contaminants on hemoglobin of larvae of aquatic midge, Chironomus riparius (Diptera: Chironomidae): A potential biomarker for ecotoxicity monitoring. Chemosphere 71(10): 1928-1936

- Hooper, H. L., R. M. Sibly, T. H. Hutchinson and S. J. Maund (2003). The influence of larval density, food availability and habitat longevity on the life history and population growth rate of the midge Chironomus riparius. Oikos 102(3): 515-524

- Keightley, P. D., R. W. Ness, D. L. Halligan and P. R. Haddrill (2013). Estimation of the spontaneous mutation rate per nucleotide site in a Drosophila melanogaster full-sib family. Genetics: genetics. 113.158758

- Keightley, P. D., U. Trivedi, M. Thomson, F. Oliver, S. Kumar and M. Blaxter (2009). Analysis of the genome sequences of three Drosophila melanogaster spontaneous mutation accumulation lines. Genome research: gr. 091231.091109

- Kelty, J. D. and R. Lee (2001). Rapid cold-hardening of Drosophila melanogaster (Diptera: Drosophiladae) during ecologically based thermoperiodic cycles. Journal of Experimental Biology 204(9): 1659-1666 
- Kraemer, C. and E. R. Schmidt (1993). The sex determining region of Chironomus thummi is associated with highly repetitive DNA and transposable elements. Chromosoma 102(8): $553-562$

- Lee, R. E., C.-p. Chen and D. L. Denlinger (1987). A rapid cold-hardening process in insects. Science 238(4832): 1415-1417

- Mathieu-Denoncourt, J., S. J. Wallace, S. R. de Solla and V. S. Langlois (2015). Plasticizer endocrine disruption: Highlighting developmental and reproductive effects in mammals and non-mammalian aquatic species. General and comparative endocrinology 219: 74-88

- Merilä, J. and A. P. Hendry (2014). Climate change, adaptation, and phenotypic plasticity: The problem and the evidence. Evolutionary Applications 7: 1-14 doi: 10.1111/eva.12137.

- Montero-Pau, J., A. Gómez and J. Muñoz (2008). Application of an inexpensive and highthroughput genomic DNA extraction method for the molecular ecology of zooplanktonic diapausing eggs. Limnology and Oceanography: Methods 6(6): 218-222

- Mukai, T. and C. C. Cockerham (1977). Spontaneous mutation rates at enzyme loci in Drosophila melanogaster. Proceedings of the National Academy of Sciences 74(6): 25142517

- Nemec, S., S. Patel, C. Nowak and M. Pfenninger (2013). Evolutionary determinants of population differences in population growth ratex habitat temperature interactions in Chironomus riparius. Oecologia 172(2): 585-594

- Neumann, D. (1962). Die anaerobiose-toleranz der larven zweier subspezies von Chironomus thummi. Zeitschrift fuer vergleichende Physiologie 46(2): 150-162 
- Nowak, C., D. Jost, C. Vogt, M. Oetken, K. Schwenk and J. Oehlmann (2007a). Consequences of inbreeding and reduced genetic variation on tolerance to cadmium stress in the midge Chironomus riparius. Aquatic Toxicology 85(4): 278-284

- Nowak, C., C. Vogt, J. B. Diogo and K. Schwenk (2007b). Genetic impoverishment in laboratory cultures of the test organism Chironomus riparius. Environmental Toxicology and Chemistry 26(5): 1018-1022

- OECD (2004). Test No. 219: Sediment-Water Chironomid Toxicity Using Spiked Water.

- OECD. (2010). "Series on Testing and Assessment No. 136 VALIDATION REPORT OF THE CHIRONOMID FULL LIFE-CYCLE TOXICITY TEST ENV/JM/MONO(2010)35." from http://www.oecd.org/officialdocuments/publicdisplaydocumentpdf/?cote=env/jm/mono(2 010)35\&doclanguage $=\mathrm{en}$.

- OECD (2011). Test No. 235: Chironomus sp., Acute Immobilisation Test.

- Oppold, A.-M., J. A. Pedrosa, M. Bálint, J. B. Diogo, J. Ilkova, J. L. Pestana and M. Pfenninger (2016). Support for the evolutionary speed hypothesis from intraspecific population genetic data in the non-biting midge Chironomus riparius. Proc. R. Soc. B 283(1825): 20152413

- Oppold, A. M. and M. Pfenninger (2017a). Direct estimation of the spontaneous mutation rate by short-term mutation accumulation lines in Chironomus riparius. Evolution Letters 1(2): 86-92 doi: doi:10.1002/evl3.8.

- Oppold, A. M., H. Schmidt, M. Rose, S. L. Hellmann, F. Dolze, F. Ripp, B. Weich, U. Schmidt-Ott, E. Schmidt and R. Kofler (2017b). Chironomus riparius (Diptera) genome 
sequencing reveals the impact of minisatellite transposable elements on population divergence. Molecular Ecology

- Oppold, A. M., H. Schmidt, M. Rose, S. L. Hellmann, F. Dolze, F. Ripp, B. Weich, U. Schmidt-Ott, E. Schmidt and R. Kofler (2017c). Chironomus riparius (Diptera) genome sequencing reveals the impact of minisatellite transposable elements on population divergence. Molecular ecology 26(12): 3256-3275

- Pedrosa, J., D. Campos, B. Cocchiararo, C. Nowak, A. M. Soares, C. Barata and J. L. Pestana (2017). Evolutionary consequences of historical metal contamination for natural populations of Chironomus riparius (Diptera: Chironomidae). Ecotoxicology 26(4): 534546

- Pfenninger, M., C. Nowak, C. Kley, D. Steinke and B. Streit (2007). Utility of DNA taxonomy and barcoding for the inference of larval community structure in morphologically cryptic Chironomus (Diptera) species. Molecular Ecology 16: 1957-1968 doi: 10.1111/j.1365-294X.2006.03136.x.

- Pinder, L. C. V. (1986). Biology of Freshwater Chironomidae. Annual Review of Entomology 31: 1-23 doi: 10.1146/annurev.en.31.010186.000245.

- Quinlan, R., M. S. Douglas and J. P. Smol (2005). Food web changes in arctic ecosystems related to climate warming. Global Change Biology 11(8): 1381-1386

- Schmidt, H., B. Greshake, B. Feldmeyer, T. Hankeln and M. Pfenninger (2013). Genomic basis of ecological niche divergence among cryptic sister species of non-biting midges. BMC genomics 14(1): 384 
- Sibley, P. K., D. A. Benoit and G. T. Ankley (1997). The significance of growth in Chironomus tentans sediment toxicity tests: Relationship to reproduction and demographic endpoints. Environmental Toxicology and Chemistry 16(2): 336-345 doi: doi:10.1002/etc.5620160232.

- T., B. E., P. R. J. O. and M. A. J. (1986). Feeding and sexual dimorphism in adult midges (Diptera: Chironomidae). Ecography 9(1): 27-32 doi: doi:10.1111/j.16000587.1986.tb01188.x.

- Taylor, E., S. Maund and D. Pascoe (1991). Toxicity of four common pollutants to the freshwater macroinvertebratesChironomus riparius Meigen (Insecta: Diptera) andGammarus pulex (L.)(Crustacea: Amphipoda). Archives of environmental contamination and toxicology 21(3): 371-376

- Vogt, C., D. Belz, S. Galluba, C. Nowak, M. Oetken and J. Oehlmann (2007a). Effects of cadmium and tributyltin on development and reproduction of the non-biting midge Chironomus riparius (Diptera)—baseline experiments for future multi-generation studies. Journal of Environmental Science and Health Part A 42(1): 1-9

- Vogt, C., C. Nowak, J. B. Diogo, M. Oetken, K. Schwenk and J. Oehlmann (2007b). Multigeneration studies with Chironomus riparius-effects of low tributyltin concentrations on life history parameters and genetic diversity. Chemosphere 67(11): 2192-2200

- Vogt, C., A. Pupp, C. Nowak, L. S. Jagodzinski, J. Baumann, D. Jost, M. Oetken and J. Oehlmann (2007c). Interaction between genetic diversity and temperature stress on lifecycle parameters and genetic variability in midge Chironomus riparius populations. Climate Research 33: 207-214 doi: 10.3354/cr033207. 
- Vogt, C., A. Pupp, C. Nowak, L. S. Jagodzinski, J. Baumann, D. Jost, M. Oetken and J. Oehlmann (2007d). Interaction between genetic diversity and temperature stress on lifecycle parameters and genetic variability in midge Chironomus riparius populations. Climate Research 33(3): 207-214

- Waldvogel, A. M., A. Wieser, T. Schell, S. Patel, H. Schmidt, T. Hankeln, B. Feldmeyer and M. Pfenninger (2018). The genomic footprint of climate adaptation in Chironomus riparius. Molecular ecology 27(6): 1439-1456

- Walker, I. R. (1987). Chironomidae (Diptera) in paleoecology. Quaternary Science Reviews 6(1): 29-40

- Watts, M. and D. Pascoe (1996). Use of the freshwater macroinvertebrate Chironomus riparius (Diptera: Chironomidae) in the assessment of sediment toxicity. Water Science and Technology 34(7-8): 101

- Weltje, L., E. Bruns and B. AG (2009). The chironomid full life-cycle test. Validation report, First draft

- Williams, K. A., D. W. Green, D. Pascoe and D. E. Gower (1986). The acute toxicity of cadmium to different larval stages of Chironomus riparius (Diptera: Chironomidae) and its ecological significance for pollution regulation. Oecologia 70(3): 362-366

- Zampicinini, G., A. Blinov, P. Cervella, V. Guryev and G. Sella (2004). Insertional polymorphism of a non-LTR mobile element (NLRCth1) in European populations of Chironomus riparius (Diptera, Chironomidae) as detected by transposon insertion display. Genome 47(6): 1154-1163 
